Pecvnia, Monográfico (2009), pp. 31-54

\title{
El inmovilizado intangible
}

\author{
Cristina Gutiérrez López \\ cristina.gutierrez.lopez@unileon.es \\ Universidad de León \\ Fac. de Ciencias Económicas y Empresariales \\ Campus de Vegazana, $\mathrm{s} / \mathrm{n}$ \\ 24071 León (España)
}

\section{CONCEPTO Y NORMATIVA}

La definición de activos intangibles se ha abordado tradicionalmente desde dos puntos de vista: aproximándose al concepto o elaborando una lista de los elementos que lo incluyen. Ambos se combinan actualmente tanto en la normativa internacional como en el caso español.

El IASB aborda la problemática contable de los activos intangibles fundamentalmente en la NIC "38. Activos inmateriales", que se modificó en marzo de 2004 a consecuencia del proyecto sobre combinaciones de negocios, que tuvo como consecuencia la versión revisada de NIC 36, NIC 38 y la nueva norma NIIF "3. Combinaciones de negocios". Las modificaciones de NIC 38 se centraban en el concepto de identificabilidad, la vida útil y 
amortización de activos intangibles, así como en la contabilización de proyectos de I+D adquiridos en una combinación de negocios ${ }^{1}$.

Por su parte, el Nuevo Plan General Contable (en adelante, NPGC) destina las normas de registro y valoración 5 y 6 a las normas generales y particulares, respectivamente. El Cuadro 1 resume los principales cambios en el tratamiento de los intangibles según el NPGC.

\section{Cuadro 1}

- Cambio de denominación: de inmovilizado inmaterial a intangible

- Criterios de reconocimiento: identificabilidad

- Activación obligatoria de gastos financieros devengados antes del funcionamiento del intangible (si su entrada en funcionamiento supera el plazo de un año)

- No hay fondo de reversión

- Los derechos sobre bienes en régimen de arrendamiento financiero no se consideran intangibles

- Pueden reconocerse beneficios en las permutas

- No todos los intangibles se amortizan: sólo si su vida útil es definida

- El fondo de comercio no se amortiza

- Intangibles con vida útil indefinida y fondo de comercio: evaluar si hay deterioro de valor y posibles reversiones posteriores del mismo (no en fondo de comercio)

- PGC Pymes: no se incluye el fondo de comercio

Las cuentas que registran los activos intangibles figuran en el subgrupo "20. Inmovilizaciones intangibles" identificándose específicamente los elementos siguientes:

200. Investigación

201. Desarrollo

202. Concesiones administrativas

203. Propiedad industrial

204. Fondo de comercio (no aparecen en el Plan de Pymes)

\footnotetext{
Por tanto, las normas NIC e interpretaciones SIC aplicables son: NIC "38. Activos inmateriales" (revisada en 2004); NIC "36. Deterioro del valor de los activos" (revisada en 2004); NIC "20. Contabilización de las subvenciones oficiales e información a revelar sobre ayudas públicas" (reordenada en 1994); NIC "23. Costes por intereses" (revisada en 1993); SIC "10. Ayudas públicas - sin relación específica con actividades de explotación"; SIC "32. Activos inmateriales costes de sitios Web".
} 
205. Derechos de traspaso

206. Aplicaciones informáticas

209. Anticipos para inmovilizado material

La amortización se registra a través de las cuentas "680. Amortización del inmovilizado intangible" (dotación del ejercicio) y "280. Amortización acumulada del inmovilizado intangible". En cuanto a la corrección por deterioro y su reversión, cuyo destino es la cuenta de pérdidas y ganancias, aparecen en las cuentas "690. Pérdidas por deterioro del inmovilizado intangible" y "790. Reversión del deterioro del inmovilizado intangible", respectivamente. El deterioro al cierre se recogerá en la cuenta de balance "290. Deterioro de valor del inmovilizado intangible". Por último, los resultados procedentes del intangible por su baja en inventario, sea cual sea el motivo, figurarán como "670. Pérdidas procedentes del inmovilizado intangible" ó "770. Beneficios procedentes del inmovilizado intangible".

\section{DEFINICIÓN Y RECONOCIMIENTO}

Siguiendo las características del subgrupo 20 del NPGC, serán activos intangibles los activos no monetarios sin apariencia física susceptibles de valoración económica. La referencia a activos no monetarios supone hablar de activos por los que no se va a recibir una cantidad determinada o determinable de dinero; es decir, no son activos financieros. La carencia de apariencia física es coherente con las consideraciones del marco conceptual, que indica que la forma física no es esencial para la existencia de un activo. Por último, su valoración económica es acorde con los criterios exigidos para el registro de activos.

El activo intangible puede mantenerse por cualquier motivo; por tanto, a diferencia del inmovilizado material, no necesita destinarse a su uso en la producción o suministro de bienes o servicios, ni a fines administrativos, ni para obtener rentas o plusvalías (caso de las inversiones inmobiliarias).

Además, de la Norma de Registro y Valoración (en adelante, NRV) 5.1 se extraen los criterios para su reconocimiento contable, a saber: 
activos

- Cumplir la definición y los criterios para el registro de

Así, serán bienes, derechos y otros recursos controlados económicamente por la empresa resultantes de sucesos pasados, de los que se espera que la empresa obtenga beneficios o rendimientos económicos en el futuro. De acuerdo a la NIC 38, dichos beneficios incluirán tanto los ingresos ordinarios procedentes de la venta de productos o servicios, como ahorros de coste ${ }^{2} \mathrm{y} / \mathrm{u}$ otros rendimientos. Respecto al control, implicará la capacidad para obtener los beneficios económicos futuros y restringir el acceso de terceros. En general, eso se materializa en un derecho legal exigible ante los tribunales, ya que el control será más difícil de demostrar en caso contrario.

Para su registro en el balance debe ser probable la obtención de dichos rendimientos, así como su valoración fiable. En este sentido, existe probabilidad cuando haya más posibilidades de que se produzcan dichos beneficios que de lo contrario, después de una evaluación basada en hipótesis razonables y fundadas. De hecho, la simple posibilidad de que la entidad identifique un valor en un activo intangible es indicativo de que considera que éste es susceptible de generar beneficios futuros, ya que en el valor atribuido se reflejan las expectativas sobre tal probabilidad. Este criterio se verifica siempre que el intangible se adquiere independientemente o como parte de una combinación de negocios. En cuanto a la valoración fiable, no arrojará dudas cuando exista una contrapartida por la adquisición del activo, sobre todo si es monetaria.

La mayor dificultad estriba, no obstante, en la posesión de los beneficios económicos futuros generados por el activo, dado que, de no haber derecho de propiedad o contrato legal sobre ellos, no existirá certeza. Así, una base de datos de clientes (no hay garantías de su continuidad futura), el capital humano (salvo que una cláusula contractual impida que abandone la entidad) o una imagen de marca valorada por la clientela (valoración que puede desaparecer posteriormente) no cumplen las condiciones para reflejarse como activos en un balance. Se trata, en estos casos, de intangibles generados internamente, que no podrán identificarse por separado, entendiendo que forman parte del fondo de

2 Así, el uso de la propiedad industrial dentro del proceso de producción puede contribuir a reducir los costes de producción futuros. 
comercio, que sólo se pondría de manifiesto mediante una adquisición onerosa.

- Ser identificable, lo que implica:

- ser separable, o "susceptible de ser separado de la empresa y vendido, cedido o entregado para su explotación, arrendado o intercambiado", o

- surgir de derechos legales o contractuales con independencia de que tales derechos sean transferibles o separables de la empresa o de otros derechos u obligaciones.

El concepto de identificabilidad está muy relacionado con las combinaciones de negocios, ya que para que un activo intangible pueda ser identificable es preciso que pueda reconocerse por separado del fondo de comercio. Por eso en NIIF-3 el fondo de comercio corresponde a los beneficios económicos futuros procedentes de activos que no han podido ser identificados individualmente y reconocidos por separado.

Los mismos requisitos se exigen a los desembolsos realizados en el activo intangible con posterioridad. Sin embargo, es difícil atribuirlos a un activo intangible y no al negocio en su conjunto, así que sólo en raras ocasiones se reconocen en el valor en libros del activo, y habitualmente se llevan al resultado.

centra en dos cuestiones:

En resumen, la problemática inicial de los intangibles de

1) ¿Se trata de activos o gastos? Para una respuesta adecuada, habrán de evaluarse las condiciones citadas antes.

2) ¿Son inmovilizados materiales o intangibles? Dado que en algunos casos ambos conceptos parecen inseparables, se deberá acudir al principio de materialidad. Así, la película grabada en una cinta de video debería reconocerse como intangible, pese al soporte físico que lo contiene.

Finalmente, no son intangibles "los gastos ocasionados con motivo del establecimiento, las marcas, cabeceras de periódicos o revistas, los sellos o denominaciones editoriales, las listas de clientes u otras partidas similares que se hayan generado internamente", entendiéndose que forman parte del fondo de comercio. 


\section{VALORACIÓN INICIAL}

De acuerdo a la NRV 5, son aplicables las normas relativas al inmovilizado material, por lo que se valorarán inicialmente, con carácter general, a precio de adquisición o coste producción. En concreto, generalizando, podemos decir que su valoración inicial se realizará al coste, que dependerá de la forma de obtención:

a) De forma independiente (adquirido en el mercado externo): precio de adquisición

b) En una combinación de negocios: valor razonable

c) De forma gratuita (subvención, donación, etc.): valor razonable

d) Intercambio de activos (permuta): normalmente, valor razonable

e) Generado internamente: coste de producción.

Los desembolsos en que se haya incurrido con relación a un activo intangible que se hubiesen reconocido inicialmente como un gasto no se reconocerán con posterioridad como parte de su coste (excepto en los generados internamente). Los desembolsos posteriores a la adquisición de un activo reconocido como intangible se incorporan como mayor valor en libros si van a permitir al activo generar beneficios económicos futuros superiores a los previstos inicialmente y puedan estimarse; en caso contrario, serán gastos del ejercicio. Se considera poco habitual que los desembolsos posteriores sean reconocidos como mayor valor en libros, ya que es más probable que representen el coste de mantener los beneficios económicos futuros esperados.

a) Activos intangibles adquiridos de forma independiente

En este caso, siempre se cumple el criterio de reconocimiento. Su valoración se efectuará con el precio de adquisición, obtenido a partir del importe facturado por el vendedor (reducido por los descuentos y rebajas) y los gastos adicionales hasta su puesta en condiciones de funcionamiento. En el NPGC, además será obligatorio (opcional en Plan 1990) incluir (también en el cálculo del coste de producción) los gastos financieros devengados hasta su puesta en condiciones de funcionamiento girados por el proveedor o asociados a financiación ajena (específica o genérica) atribuible a la adquisición, en aquellos 
inmovilizados que necesiten más de un año para estar en condiciones de uso $^{3}$ (denominados por las NIC "activos cualificados").

En caso de que dicha adquisición se haya producido a crédito, surgirán pasivos financieros (NRV 9), débitos por operaciones no comerciales, que deberán valorarse inicialmente a valor razonable (precio de la transacción o valor razonable de la contraprestación recibida ajustado por los costes de la transacción) y posteriormente a coste amortizado.

Ejemplo n ${ }^{0}$ 1.- Adquisición de inmovilizado intangible con pago aplazado

A finales del ejercicio X1 una entidad adquiere un software de gestión por 3.000€, que pagará a través de dos cuotas dentro de 1 y 2 años de 1.700 y 2.000€, respectivamente.

Solución.-

Finales X1. Compra del intangible:

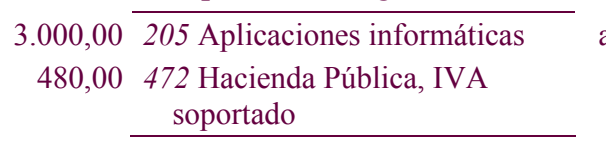
173 Proveedores de inmovilizado a largo plazo

Valoración posterior: coste amortizado

Cálculo del TIE:

$$
3.480=\frac{1.700}{(1+i)}+\frac{2.000}{(1+i)^{2}} \rightarrow i=\mathrm{TIE}=4,0727 \%
$$

\begin{tabular}{|c|c|c|c|c|}
\hline Período & Intereses & Pagos & $\begin{array}{c}\text { Amortización } \\
\text { principal }\end{array}$ & Coste amortizado \\
\hline- & & & & 3.480 \\
\hline 1 & $i \times 3.480=141,7323$ & 1.700 & $1.558,2677$ & $3.480-1700+141,7323=1.921,7323$ \\
\hline 2 & $i \times 1.921,7323=78,2677$ & 2.000 & $1.921,7323$ & - \\
\hline & 220 & 3.700 & 3.480 & \\
\hline
\end{tabular}

3 Según la NIC 23, el tratamiento preferente de los gastos financieros será llevarlos al resultado del ejercicio, si bien se permite su activación previo cumplimiento de ciertos requisitos. Si el préstamo es financiación específica para la adquisición/fabricación del activo, los costes por intereses susceptibles de capitalización se obtendrán por diferencia de los costes reales en que se ha incurrido durante el ejercicio en relación con ese préstamo menos los rendimientos procedentes de la financiación no aplicada al activo, cuando esos fondos se hayan destinado temporalmente a inversiones financieras.

En cuanto a las diferencias de cambio, el NPGC no dice nada al respecto, pero según las NIC no está permitida su inclusión. 
Finales X2:

\begin{tabular}{|c|c|c|c|c|}
\hline & & \\
\hline 141,7323 & 662 Intereses de deudas & a & $\begin{array}{l}173 \text { Proveedores de inmovilizado } \\
\text { a largo plazo }\end{array}$ & 141,7323 \\
\hline $1.700,00$ & $\begin{array}{l}173 \text { Proveedores de inmovilizado } \\
\text { a largo plazo }\end{array}$ & a & 57 Tesorería & $1.700,00$ \\
\hline $1.921,7323$ & $\begin{array}{l}173 \text { Proveedores de inmovilizado } \\
\text { a largo plazo }\end{array}$ & a & $\begin{array}{l}523 \text { Proveedores de inmovilizado } \\
\text { a corto plazo }\end{array}$ & $1.921,7323$ \\
\hline \multicolumn{5}{|l|}{ Finales X3: } \\
\hline 78,2677 & 662 Intereses de deudas & $\mathrm{a}$ & $\begin{array}{l}523 \text { Proveedores de inmovilizado } \\
\text { a corto plazo }\end{array}$ & 78,2677 \\
\hline $2.000,00$ & $\begin{array}{l}523 \text { Proveedores de inmovilizado } \\
\text { a corto plazo }\end{array}$ & a & 57 Tesorería & $2.000,00$ \\
\hline
\end{tabular}

b) Adquiridos en una combinación de negocios

Se reconocen como activo intangible (independientemente de que estuvieran reconocidos como tal en las cuentas anuales de la entidad adquirida antes de la combinación de negocios) si cumplen la definición de activo intangible (identificabilidad) y su valor razonable se puede estimar fiablemente.

Como señala la NIC 38 , el requisito de reconocimiento de beneficios económicos futuros se cumple siempre, ya que el valor razonable refleja las expectativas del mercado de la probabilidad de que tales beneficios fluyan a la entidad ${ }^{4}$. Normalmente esta cifra puede valorarse de forma fiable para reconocerse por separado del fondo de comercio; no obstante, si existe un rango de posibles resultados con probabilidades distintas, se considera que esa incertidumbre forma parte de la fijación del valor y no demuestra imposibilidad de determinarlo de forma fiable. Si un activo intangible adquirido así tiene una vida útil definida, se presume que su valor razonable puede determinarse con suficiente fiabilidad.

Siguiendo la citada norma internacional, se establece el siguiente rango de precios para el valor razonable:

4 Los criterios de reconocimiento de los activos intangibles procedentes de combinaciones de empresas no incorporan el criterio de probabilidad existente en los criterios generales de reconocimiento de los activos intangibles, ya que usan el valor razonable para la valoración. 
- Cuando existe mercado activo, el precio de cotización es la estimación más razonable, aunque es poco común que exista un mercado activo ${ }^{5}$.

- No existe mercado activo: precio de la transacción similar más reciente.

- Valor razonable en activos intangibles identificables, pero no separables: en general, para reconocer un activo intangible de forma independiente al fondo de comercio adquirido, su valor debe medirse con fiabilidad. Sin embargo, puede que pese a ser identificable y adquirirse de forma independiente, el intangible deba ir unido a un activo material. En estos casos, el adquirente reconocerá el grupo de activos como un activo único independientemente del fondo de comercio. Así se actuará si los valores razonables de esos activos no puedan medirse con fiabilidad.

- Aplicación de técnicas de estimación.

\section{c) Obtención gratuita}

De acuerdo a la NRV "18. Subvenciones, donaciones y legados recibidos", las subvenciones, donaciones y legados recibidos no reintegrables se valorarán por su valor razonable, y se contabilizarán como ingresos directamente imputados a patrimonio neto ${ }^{6}$. Su traspaso a resultados del ejercicio, de acuerdo a su finalidad (adquisición de inmovilizados intangibles, materiales o inversiones inmobiliarias) se hará en proporción a la dotación de amortizaciones o, en su caso, cuando se produzca su enajenación, corrección valorativa por deterioro o baja en balance.

5 Un mercado activo cumple las siguientes condiciones: a) los bienes/servicios intercambiados son homogéneos, b) se pueden encontrar en todo momento compradores y vendedores para un determinado bien/servicio, c) los precios están disponibles para el público.

6 En la normativa internacional (NIC 20) se establecen dos alternativas para el tratamiento de las subvenciones: a) Ingresos diferidos que se irán imputando a resultados a lo largo de la vida útil de los correspondientes activos; por tanto, la subvención se reconocerá en la cuenta de resultados como un ingreso sobre una base sistemática y racional. La devolución de la subvención se reconocería reduciendo el saldo de dicha partida de ingresos diferidos por el importe a reembolsar. b) Deducir el importe de la subvención del valor de los activos con los que se relaciona; se reconocerán en la cuenta de resultados a lo largo de la vida útil de los activos reduciendo la dotación anual por la amortización de los activos; por tanto, la subvención afectará al resultado como un cargo menor en concepto de dotación anual por la depreciación del activo. Posteriormente, la devolución de la subvención se reconocerá registrando como contrapartida un aumento del importe en libros del activo correspondiente; la amortización acumulada que podría haberse cargado adicionalmente hasta la fecha, en ausencia de la subvención, se carga inmediatamente a resultados. 


\section{Ejemplo n ${ }^{\circ}$ 2.- Inmovilizado intangible recibido en donación}

Una empresa ha recibido a principios de año y de forma gratuita de un tercero un novedoso programa informático, que utilizará durante 8 años, período durante el que se estima permitirá obtener flujos de efectivo. El valor razonable del software es de $40.000 €$.

Solución (prescindiendo del efecto impositivo).-

\section{1) Plan General Contable}

Recepción del software:

40.000,00 205 Aplicaciones informáticas

A Aplicaciones informaticas

31-12 Depreciación del software:

\begin{tabular}{cc}
$5.000,00$ & $\begin{array}{c}680 \text { Amortización del } \\
\text { inmovilizado intangible } \\
40.000 / 8=5.000\end{array}$ \\
\hline
\end{tabular}

31-12 Traspaso al resultado del ejercicio:

$$
\begin{gathered}
5.000,00 \\
841 \text { Transferencias de donaciones } \\
\text { y legados de capital }
\end{gathered}
$$

a 280 Amortización acumulada del inmovilizado intangible

$5.000,00$

941 Ingresos de donaciones y
legados de capital

-

76 Subvenciones, donaciones y legados de capital transferidos

31-12 Traspaso al estado de cambios en patrimonio neto:

\begin{tabular}{cc}
$40.000,00$ & $\begin{array}{l}941 \text { Ingresos de donaciones y } \\
\text { legados de capital }\end{array}$ \\
\cline { 2 - 2 } $5.000,00$ & $\begin{array}{l}131 \text { Donaciones y legados de } \\
\text { capital }\end{array}$ \\
\hline
\end{tabular}
a 131 Donaciones y legados de capital
a 841 Transferencias de donaciones
y legados de capital al resultado del ejercicio

Estado de cambios en el patrimonio neto:
a) Resultado de la cuenta de pérdidas y ganancias
b) Ingresos y gastos imputados directamente en el patrimonio neto
c) Transferencias a la cuenta de pérdidas y ganancias $5.000,00$
Total ingresos y gastos reconocidos $35.000,00$

\section{2) Plan Pymes}

Recepción del software:

40.000,00 205 Aplicaciones informáticas

131 Donaciones y legados de
capital

31-12 Depreciación del software:

\begin{tabular}{cc}
$5.000,00$ & $\begin{array}{c}680 \text { Amortización del } \\
\text { inmovilizado intangible } \\
40.000 / 8=5.000\end{array}$ \\
\hline
\end{tabular}
280 Amortización acumulada del inmovilizado intangible


31-12 Traspaso al resultado del ejercicio:

$\begin{array}{cccc}5.000,00 & \begin{array}{c}131 \text { Donaciones y legados de } \\ \text { capital }\end{array} & \begin{array}{c}746 \text { Subvenciones, donaciones y } \\ \text { legados de capital transferidos } \\ \text { al resultado del ejercicio }\end{array}\end{array}$

$5.000,00$

\section{d) Permuta}

En caso de intercambio de activos, siguiendo las normas del inmovilizado material y el supuesto planteado al respecto, el elemento entregado se dará de baja por su valor contable o valor en libros. En cuanto al elemento recibido, su valoración dependerá del tipo de permuta.

En caso de permutas comerciales, por el valor razonable del entregado, o el del recibido, si es más fiable, con el límite de este último. En caso de permutas no comerciales (o si se desconocen los valores razonables), se empleará el valor contable del entregado.

La cuestión estriba pues en distinguir la naturaleza de la permuta. Será comercial si la configuración de los flujos de efectivo de ambos activos difiere, o el valor actual de los flujos de efectivo de las actividades empresariales afectadas por la operación se ve modificada, y en esos casos, las diferencias sean significativas en comparación con el valor razonable de dichos activos ${ }^{7}$.

Por tanto, se admite la posibilidad de reconocer beneficios en las permutas, dado el cambio en la redacción del principio de prudencia, que pasa de "beneficios realizados" ${ }^{18}$ a "obtenidos", si bien estos estarán limitados por el valor razonable del elemento recibido, dado que actúa como límite. Aunque el NPGC no es explícito al respecto, según las NIC, no es necesario que la entidad realice cálculos detallados para decidir si la permuta es comercial o no, sino que basta con estimaciones. Además, de acuerdo a la normativa internacional, los beneficios surgidos de la operación pueden ser superiores, al no fijarse un valor razonable como límite.

$7 \quad$ En el Borrador del NPGC se establecía que en ningún caso serían permutas comerciales aquellas de activos de la misma naturaleza y uso. Sin embargo, esta redacción no se ha mantenido en el NPGC, tal y como se recoge en las NIC, que eliminaron de anteriores versiones de la NIC "16. Inmovilizado material" toda alusión al término "activos similares", entendiendo que activos de la misma naturaleza, e incluso del mismo uso, pueden dar lugar a diferentes expectativas en la configuración de los flujos de efectivo.

8 Es decir, sin que se produzca liquidación o venta. Es el caso también de cambios de valor en ciertos activos/pasivos por cambios en el valor razonable. 
e) Intangible generado internamente

El criterio de valoración será el coste de producción, formado por el precio de adquisición de los materiales y otros ${ }^{9}$, los costes directos, costes indirectos (si corresponden al período de fabricación/ construcción y son necesarios para la puesta del activo en condiciones de funcionamiento), impuestos indirectos no recuperables y los gastos financieros en los supuestos mencionados anteriormente.

En algunos casos, el principal problema que surge en el reconocimiento de este tipo de activos reside en la dificultad para estimar fiablemente su coste, así como en determinar el momento en que el activo cumple la característica de identificabilidad y se espera que genere de forma probable beneficios económicos ${ }^{10}$. Es el caso de los gastos de I+D.

El fondo de comercio generado internamente no se reconocerá como un activo.

\section{VALORACIÓN POSTERIOR}

En general, los gastos ocasionados por un activo inmaterial después de su adquisición o finalización no se activan (se registran como gasto), salvo que:

- formen parte del coste de un activo intangible que cumpla los criterios de reconocimiento, o

- la partida haya sido adquirida en una combinación de negocios, y no pueda ser reconocida como un activo intangible. En este caso, esa cifra formará parte del importe atribuido al fondo de comercio en la fecha de adquisición (NRV "19. Combinaciones de negocios").

9 El NPGC no ha aprovechado la ocasión para referirse al coste de los materiales empleados, que no necesariamente ha de coincidir con los comprados.

10 El término "probable" no está definido con relación a los activos intangibles, pero eso no significa que deba estar garantizado que un proyecto alcance el éxito antes de capitalizar cualquier gasto de desarrollo. Así, una entidad farmacéutica no debería considerar como gastos todos los costes por desarrollo, sólo porque exista una posibilidad de que no se apruebe la comercialización de los nuevos medicamentos por parte de la autoridad. Debería hacerse una evaluación de la probabilidad de éxito de cada caso individual. 
En un momento posterior deberá considerarse la amortización y el deterioro de valor:

\section{- Amortización}

Deberá estimarse si el intangible tiene una vida útil definida o indefinida, tal que en el segundo caso no haya un límite previsible para el período en que el activo genere entradas de efectivo para la entidad. Aquellos intangibles con vida útil indefinida no se amortizarán, aunque deberá estimarse su posible deterioro de valor al menos al cierre de cada ejercicio. Además, su vida útil deberá revisarse para comprobar que sigue manteniendo dicha calificación ${ }^{11}$. En caso de cambios en la vida útil (así como en el método de amortización o en el valor residual), se aplicará lo establecido en la NRV "22. Cambios en criterios contables, errores y estimaciones contables", sobre cambios en estimaciones contables, de aplicación prospectiva. También deberá ajustarse la amortización en caso de producirse deterioros de valor.

A efectos prácticos, si una entidad tiene una concesión administrativa que da derecho a explotar la cafetería de la universidad durante 5 años, la vida útil será definida y el elemento deberá amortizarse. Por el contrario, si ha obtenido la patente de un nuevo diseño, que le otorga el derecho a explotarla por tiempo indefinido, no se amortizará en tanto la vida útil no sea por un período temporal determinado.

La amortización deberá determinarse de una forma sistemática y racional, según la vida útil y el valor residual ${ }^{12}$, considerando tanto la depreciación funcional como la física, así como la obsolescencia.

Mientras que el NPGC no se decanta por ningún método de amortización determinado, según la NIC-38 éste debería reflejar el patrón de consumo esperado de los beneficios económicos futuros derivados del activo, presumiendo que debería ser lineal, ya que la cifra resultante de la aplicación de otro método normalmente no será inferior a la calculada según el método lineal.

11 Que existan dificultades para determinar la vida útil no significa que un activo intangible tenga una vida útil indefinida, pero tampoco que su vida sea corta de forma poco realista.

12 Según la NIC 38, se supone que el valor residual es nulo, salvo que a) exista compromiso de un tercero para comprar el activo al final de su vida útil, b) exista un mercado activo para ese activo intangible y pueda determinarse el valor residual con referencia a ese mercado y sea probable que el mercado subsista el final de la vida útil del mismo. 


\section{- Deterioro de valor}

Los activos intangibles con vida útil indefinida no se amortizan sino que se someten a pruebas de deterioro de valor en cada fecha de cierre. Dicho deterioro se producirá si el importe recuperable es inferior al valor en libros. El importe recuperable será el mayor de:

- Valor razonable menos costes de venta ${ }^{13}, 0$

- Valor en uso ${ }^{14}$.

Además, posteriormente habrá que analizar la posible reversión del deterioro de valor si cambian las estimaciones para hallar el importe recuperable. En este caso, deberá aumentarse el valor contable del activo hasta dicho valor recuperable, con el límite del valor contable que estaría reconocido en la fecha de reversión si no se hubiera reconocido pérdida por deterioro previamente.

\section{Ejemplo no 3.- Deterioro de un activo}

Una empresa tiene una patente de diseño de un ordenador que compró el 1-1-X0 por $68.000 €$, a la que se le estima una vida útil de 5 años y un valor residual de 6.000€. Para calcular su amortización, aplica el método lineal.

A 31-12-X2 se dispone a elaborar sus estados financieros y observa que el ordenador que se fabrica con esa patente se está quedando obsoleto al lanzar la competencia otro equipo informático con gran aceptación en el mercado.

La última transacción conocida de un elemento similar se realizó a 22.000€, y además la empresa determina su valor de uso para ver si existe deterioro de valor. El tipo de descuento estimado es del 8,5\%. Considera que los flujos futuros en los dos años restantes serán 14.000 y $12.000 €$ respectivamente.

Para la solución se consideran tres alternativas:

13 Según la NIC 36, el valor razonable dependerá de las circunstancias del activo: si la empresa tiene un compromiso formal de venta, será el precio pactado con el comprador, mientras que si el activo se negocia en un mercado activo, será el precio del mercado activo (precio comprador corriente), y en caso de no existir ni compromiso formal de venta ni mercado activo podrá emplearse el valor de transacciones recientes con activos similares en el mismo sector industrial.

14 Dependerá de la situación del activo que su mayor valor recuperable sea uno u otro. Por ejemplo, es poco probable que un activo no corriente destinado a la venta genere valor a través de su utilización, y en cambio es posible que la empresa pueda conseguirlo a través de su venta, obteniendo el importe equivalente a valor razonable menos costes de venta. Sin embargo, tratándose de un inmovilizado que la entidad destina a su actividad productiva, seguramente se recupere un mayor valor a través de su uso que si se vendiera. 
a) Valorar y registrar contablemente, si es necesario, el deterioro de valor del activo.

b) Suponer que, al no existir un mercado activo de patentes de ordenador, la empresa no tiene valor razonable de referencia y estima su valor de uso, con un tipo de descuento del 8,5\%, considerando los siguientes flujos esperados, con sus respectivas probabilidades:

\begin{tabular}{|c|c|c|c|}
\hline Año & $\begin{array}{c}\text { Escenario favorable } \\
\mathbf{2 0 \%}\end{array}$ & $\begin{array}{c}\text { Normal } \\
\mathbf{6 5 \%}\end{array}$ & $\begin{array}{c}\text { Desfavorable } \\
\mathbf{1 5 \%}\end{array}$ \\
\hline $\mathrm{X} 3$ & 20.000 & 16.000 & 13.000 \\
\hline $\mathrm{X} 4$ & 19.500 & 14.000 & 10.000 \\
\hline
\end{tabular}

c) Con los datos del apartado a) anterior, durante el ejercicio X3 la empresa entiende que la disminución de valor del ejercicio anterior fue un hecho puntual que no se volverá a repetir. Los flujos de efectivo para el año restante de vida del elemento (X4) son 28.000€. Valoración al cierre.

\section{Solución.-}

a) Cálculo del valor en uso:

\begin{tabular}{|c|c|c|}
\hline Año & $\begin{array}{c}\text { Flujos de efectivo } \\
\text { netos anuales }\end{array}$ & $\begin{array}{c}\text { Valor actual de los } \\
\text { flujos de efectivo }\end{array}$ \\
\hline X3 & 14.000 & $14.000(1+0,085)^{-1}=12.903,22$ \\
\hline X4 & 12.000 & $12.000(1+0,085)^{-2}=10.193,46$ \\
\hline Total & & $23.096,68$ \\
\hline
\end{tabular}

Por tanto, a 31-12-X2:

- Valor contable

Precio de adquisición - Amortización acumulada:

$$
68.000-\left(\frac{68.000-6.000}{5} 3\right)=68.000-37.200=30.800
$$

- Importe recuperable: Mayor de

Valor razonable - costes de venta $=\ldots \ldots \ldots \ldots \ldots \ldots \ldots . .22 .000,00$

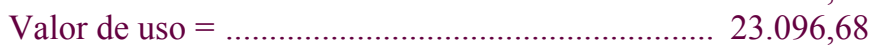

- $\quad$ Deterioro de valor $=30.800-23.096,68=\ldots \ldots \ldots \ldots . \quad 7.703,32$

31-12-X2 Registro de la pérdida por deterioro:

\begin{tabular}{|c|c|c|}
\hline $.703,32$ & $\begin{array}{l}690 \text { Pérdidas por deterioro del } \\
\text { inmovilizado intangible }\end{array}$ & \\
\hline
\end{tabular}

Después de reconocer una pérdida por deterioro, el cargo por depreciación del activo debe ajustarse en los ejercicios futuros para distribuir el importe en libros revisado del activo (es decir, valor contable), de forma sistemática durante su vida útil restante.

A partir de entonces, el cálculo de la amortización para los ejercicios X3 y X4 será $23.096,68 / 2=11.548,34$ 
b) Cálculo del valor en uso:

\begin{tabular}{|c|c|c|}
\hline Año & $\begin{array}{c}\text { Flujos de efectivo } \\
\text { netos anuales }\end{array}$ & $\begin{array}{c}\text { Valor actual de los } \\
\text { flujos de efectivo }\end{array}$ \\
\hline X3 & $(20 \% 20.000)+(65 \% 16.000)+(15 \% 13.000)=16.350$ & $16.350(1+0,085)^{-1}=15.069,12$ \\
\hline X4 & $(20 \% 19.500)+(65 \% 14.000)+(15 \% 10.000)=14.500$ & $14.500(1+0,085)^{-2}=12.317,10$ \\
\hline Total & & $27.386,22$ \\
\hline
\end{tabular}

Por tanto, a 31-12-X2:

- Valor contable:

Precio de adquisición - Amortización acumulada . $\quad 30.800$

- Importe recuperable: Mayor de

Valor razonable - costes de venta: ....................... No existe

Valor de uso …............................................. 27.386,22

- Deterioro de valor .............................................. $3.413,78$

31-12-X2 Registro contable:

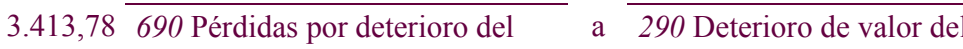
inmovilizado intangible inmovilizado intangible

c)

\begin{tabular}{|c|c|c|}
\hline & 31-12-X2 & 31-12-X3 \\
\hline $\begin{array}{c}\text { Valor contable } \\
\text { (después del deterioro) }\end{array}$ & $68.000-37.200-7.703,32=23.096,68$ & $\begin{array}{c}\text { Valor Contable 31-12-X2 - Amortiz.X3 } \\
23.096,68-(23.096,68 / 2)=11.548,34\end{array}$ \\
\hline $\begin{array}{l}\text { Valor contable } \\
\text { (antes del deterioro) }\end{array}$ & $68.000-37.200=30.800$ & $\begin{array}{l}68.000-[(68.000-6.000) \times 4 / 5]= \\
68.000-49.600=18.400 \text { Límite }\end{array}$ \\
\hline $\begin{array}{l}\text { Importe recuperable: } \\
\text { Valor en uso }\end{array}$ & $23.096,68$ & $28.000(1+0,085)^{-1}=25.806,45$ \\
\hline Deterioro & $\begin{array}{l}30.800-23.096,68=7.703,32 \\
\text { Valor contable }>\text { Valor en uso }\end{array}$ & $\begin{array}{c}\text { No hay deterioro } \\
25.806,45>11.548,34 \\
\text { Valor en uso }>\text { Valor contable }\end{array}$ \\
\hline
\end{tabular}

La contabilización de la reversión del deterioro no puede superar el valor contable que tendría el elemento de no haber existido el deterioro (18.400€) y por tanto la reversión será de $6.815,66 €$ y no de $14.258,11 €(25.806,45-11.548,34)$.

6.851,66 290 Deterioro de valor del a 790 Reversión del deterioro del inmovilizado intangible inmovilizado intangible $6.851,66$

Valor contable $=68.000-37.200-7.703,32-11.548,34+6.851,66=18.400$

En caso de reversión del deterioro de un activo, la empresa debe revisar si la vida útil restante, el método de amortización o el valor residual deben ser ajustados.

Además de la amortización y el deterioro de valor, que determinan la valoración posterior de los intangibles al coste (esto es, el 
coste histórico reducido por la amortización acumulada y/o el deterioro de valor), según las normas internacionales es posible también aplicar el modelo de revalorización, según el cual la valoración posterior coincidirá con el valor revalorizado (valor razonable) menos la amortización acumulada $\mathrm{y} / \mathrm{o}$ el deterioro de valor.

En este caso, al aumentar el valor en libros, se reflejarán reservas de revalorización (salvo que previamente se hubieran producido pérdidas, en cuyo caso procederá primero registrar beneficios en la cuenta de pérdidas y ganancias, para posteriormente reconocer las reservas por la parte restante, si la hubiera), y si disminuye se reconocerán pérdidas (salvo que previamente hubiera habido una revalorización, procediendo a cancelar la reserva en primer lugar).

\section{BAJA EN INVENTARIO}

Se reconocerá la baja de un activo intangible cuando:

a) Se enajene

b) No se espere obtener rendimientos económicos futuros de su utilización o enajenación.

En estos casos, se cancelará por su valor contable, pudiendo producirse resultados en la operación. En caso de ventas a crédito, el derecho será un activo financiero, registrándose inicialmente a valor razonable y posteriormente a coste amortizado. Se aplican pues los mismos criterios que indican las normas para inmovilizados materiales.

\section{NORMAS PARTICULARES}

\section{Gastos de I+D}

El criterio general para el registro de los gastos de investigación y desarrollo es considerarlos gastos del ejercicio en que se asumen. No obstante, si se cumplen ciertas condiciones (las mismas del Plan 1990), podrán o deberán activarse, según el caso. Dichos requisitos serán:

- Están individualizados por proyectos y su coste claramente especificado para distribuirse en el tiempo. 
- Hay motivos fundados de éxito técnico y rentabilidad económicocomercial del proyecto.

Desde que se cumplan estas exigencias, los gastos de investigación "podrán activarse", y los de desarrollo "se reconocerán en el activo"15 (NRV 6.a). Por tanto, mientras que en el Plan 1990 la posibilidad de activar los gastos era voluntaria en ambos casos (radicando pues las diferencias entre ambos conceptos en su definición y en el punto de inicio para el cálculo de amortizaciones), en el NPGC dicha opción se limita a la investigación, mientras que en el caso del desarrollo es obligatoria.

A partir de entonces, deberán ser amortizados durante su vida útil, y siempre dentro del plazo de cinco años, que podrá ampliarse en caso de gastos de desarrollo con la correspondiente explicación en la memoria. La redacción de la NRV 6.a) no deja claro cuál es el momento en que debe iniciarse el cálculo de la amortización, por lo que es posible aplicar la Resolución del ICAC sobre inmovilizado material del 21-1-92, según la cual la amortización de los gastos de investigación se realizará a partir del ejercicio en que se activen, y para los de desarrollo a partir de la fecha de finalización del proyecto ${ }^{16}$.

\section{Ejemplo n ${ }^{0}$ 4.- Gastos de investigación}

Una entidad decide llevar a cabo directamente la investigación necesaria para el desarrollo de un proyecto que permita optimizar su proceso de fabricación. Para ello asume los siguientes gastos en los ejercicios X1, X2 y X3, considerando ya a finales del primer año que hay motivos para esperar el éxito técnico y la rentabilidad del proyecto, decidiendo su activación. Asimismo, se emplea el plazo máximo de amortización establecido por el PGC.

\begin{tabular}{|l|c|c|c|}
\hline & $\boldsymbol{X} \mathbf{1}$ & $\boldsymbol{X} \mathbf{2}$ & $\boldsymbol{X} \mathbf{3}$ \\
\hline Mano de obra & 12.000 & 9.000 & 8.900 \\
\hline Materiales & 3.500 & 2.000 & 1.500 \\
\hline Amortización equipos & 2.000 & 2.100 & 2.050 \\
\hline Total & 17.500 & 13.100 & 12.450 \\
\hline
\end{tabular}

nunca activarse

15 Sin embargo, de acuerdo a la NIC 38, los gastos de investigación no podrán

16 Como se indica en la Introducción al NPGC, hasta que se produzcan los cambios en las adaptaciones sectoriales y las Resoluciones emitidas por el ICAC, esas normas siguen vigentes salvo que se opongan expresamente a los nuevos criterios contenidos en el NPGC. 


\section{Solución.-}

Registro contable en los ejercicios X1, X2 y X3:

Ejercicio X1:
$12.000 \quad 64$ Gastos de personal
a $\overline{281 \text { Amortización acumulada del }}$
3.50060 Compras inmovilizado material
2.000
2.000681 Amortización del
a Tesorería/Pasivos
inmovilizado material

Activación de gastos de investigación:
17.500200 Investigación

a 730 Trabajos realizados para el inmovilizado intangible

Ejercicio X2

$9.000 \quad 64$ Gastos de personal

2.00060 Compras

2.100681 Amortización del

inmovilizado material a 281 Amortización acumulada del inmovilizado material

2.100

a Tesorería/Pasivos

Activación de gastos de investigación:
13.100200 Investigación

a

730 Trabajos realizados para el
inmovilizado intangible

El cálculo de la amortización para cada ejercicio será:

\begin{tabular}{|c|c|c|c|c|}
\hline \multirow[b]{2}{*}{ Ejercicio } & \multicolumn{3}{|c|}{ Amortización de gastos capitalizados en... } & \multirow{2}{*}{$\begin{array}{c}\text { Amortización } \\
\text { del ejercicio }\end{array}$} \\
\hline & X1 & X2 & X3 & \\
\hline X1 & - & - & - & - \\
\hline $\mathrm{X} 2$ & 3.500 & - & - & 3.500 \\
\hline $\mathrm{X} 3$ & 3.500 & 2.620 & - & 6.120 \\
\hline $\mathrm{X} 4$ & 3.500 & 2.620 & 2.490 & 8.610 \\
\hline $\mathrm{X} 5$ & 3.500 & 2.620 & 2.490 & 8.610 \\
\hline X6 & 3.500 & 2.620 & 2.490 & 8.610 \\
\hline $\mathrm{X} 7$ & & 2.620 & 2.490 & 5.110 \\
\hline $\mathrm{X} 8$ & & & 2.490 & 2.490 \\
\hline Total & 17.500 & 13.100 & 12.450 & 43.050 \\
\hline
\end{tabular}

31-12-X2 Amortización:

\begin{tabular}{c}
$3.500 \begin{array}{c}680 \text { Amortización del } \\
\text { inmovilizado intangible }\end{array}$ \\
\hline
\end{tabular}
280 Amortización acumulada del inmovilizado intangible

Ejercicio X3:

\begin{tabular}{|c|c|c|c|}
\hline $\begin{array}{l}8.900 \\
1.500\end{array}$ & $\begin{array}{l}64 \text { Gastos de personal } \\
60 \text { Compras }\end{array}$ & $\mathrm{a}$ & $\begin{array}{l}281 \text { Amortización acumulada del } \\
\text { inmovilizado material }\end{array}$ \\
\hline 2.050 & $\begin{array}{l}681 \text { Amortización del } \\
\text { inmovilizado material }\end{array}$ & a & Tesorería/Pasivos \\
\hline
\end{tabular}


Activación de gastos de investigación:

$$
1 2 . 4 5 0 \longdiv { 2 0 0 \text { Investigación } }
$$

730 Trabajos realizados para el
inmovilizado intangible

280 Amortización acumulada del inmovilizado intangible

\section{Otros intangibles}

En el NPGC también se incluyen como activos intangibles los gastos de desarrollo de páginas Web, debido a la influencia de la SIC "32. Activos inmateriales. Costes de sitios Web", que considera que el sitio Web, propiedad de una empresa, que surge del desarrollo y está disponible para acceso interno o externo, es un activo inmaterial generado internamente que está sujeto a los requerimientos de la NIC 38.

De este modo, una empresa puede incurrir en desembolsos para el desarrollo y mantenimiento de su sitio Web, ya sea para acceso interno (empleado para contener información sobre las políticas de la compañía y los detalles de clientes, así como para buscar información relevante) o externo (propósitos como la promoción y el anuncio de los productos y servicios de la empresa, prestación de servicios electrónicos o la venta de productos y servicios).

En el caso de las concesiones administrativas ${ }^{17}$, se amortizarán de forma sistemática durante un plazo que no podrá superar el período concesional. Así, tal y como establece el marco conceptual, en activos sometidos a reversión su vida útil será el período concesional cuando éste sea inferior a la vida económica del activo, entendiéndose por esta última el periodo durante el cual el activo sea utilizable o el

17 Sin embargo, de acuerdo al IFRIC "12. Concesiones de servicios públicos", sólo se reconocerán como intangibles aquellos contratos de concesión en los que la empresa tenga el derecho al acceso, sometiéndose al riesgo de demanda (cobrando un precio a los usuarios que utilicen esa infraestructura) aún cuando la Administración garantice el equilibrio económico-financiero de la concesión complementando los ingresos obtenidos o ampliando el período de concesión; por el contrario, si la empresa concesionaria obtiene un derecho incondicional a recibir tesorería de la Administración, se tratará de un inmovilizado financiero. La aprobación de la norma por el derecho comunitario implicaría que las empresas concesionarias españolas utilicen criterios distintos para sus cuentas consolidadas (que recogerán importantes pérdidas, dado que no podrán capitalizar los costes financieros a partir del momento de clasificación del activo como intangible) e individuales, al menos a la espera de la aprobación de la adaptación sectorial correspondiente. 
número de unidades de producción que se espera obtener de él. Desaparece, respecto al Plan 1990, el fondo de reversión.

\section{Fondo de comercio}

Se trata del exceso del coste de adquisición sobre el valor de la participación del comprador en el valor razonable de los activos y pasivos identificables adquiridos.

En el NPGC el fondo de comercio no se amortizará, aunque su posible deterioro deberá analizarse al menos al final de cada ejercicio (no siendo posible su reversión, dado que esta sería un incremento de valor, que como intangible generado internamente no puede activarse). Su falta de amortización se deriva de la NIIF 3, que no considera su depreciación sistemática. Este cambio modifica las condiciones para la distribución de beneficios: ahora es preciso dotar sistemáticamente una reserva indisponible por un importe igual al fondo de comercio que figura en el activo, para lo que habrá que destinar una cifra del beneficio (o en su defecto, de las reservas de libre disposición) que represente al menos el $5 \%$ del fondo de comercio que aparezca en el balance.

Por otro lado, considerando que el fondo de comercio no genera entradas de efectivo de forma independiente de otros activos o grupos de activos, no se comprueba su deterioro de forma independiente, sino que su importe debe distribuirse entre las UGE (Unidades Generadoras de Efectivo) del adquirente que se espera se vean beneficiadas por las sinergias de la combinación de negocios, entendiéndose por UGE el grupo identificable de activos más pequeño, que genera entradas de efectivo a favor de la entidad que son independientes de los flujos de efectivo derivados de otros activos o grupos de activos.

Así, si se produce el deterioro de valor de una UGE, su distribución se realizará con el orden siguiente:

1. Se reduce el fondo de comercio atribuido a la UGE. Se trata pues de un caso excepcional, en que el deterioro se registra directamente contra la cuenta de activo que representa al fondo de comercio.

2. Se reduce el resto de los activos, prorrateando el valor en libros de cada uno, siempre que su valor en libros no sea después inferior al mayor de: 

a) Valor razonable menos Costes de venta
b) Valor en uso
c) Cero

\section{Ejemplo $n^{0}$ 5.- Deterioro de valor de una unidad generadora de efectivo}

La sociedad Prueba había adquirido a la sociedad Fondo, considerada como una única UGE. Prueba tiene indicios de que debe corregirse el valor de Fondo, estimando que el valor recuperable de ésta son 600 um. Los datos del Fondo son:

\begin{tabular}{|l|c|c|c|}
\hline & $\begin{array}{c}\text { Importe } \\
\text { en libros }\end{array}$ & $\begin{array}{c}\text { Precio neto } \\
\text { de venta }\end{array}$ & $\begin{array}{c}\text { Valor } \\
\text { en uso }\end{array}$ \\
\hline Fondo de comercio & 300 & 0 & \\
\hline $\begin{array}{l}\text { Inmovilizado material } \\
\quad \begin{array}{l}\text { Maquinaria } \\
\text { Edificio }\end{array}\end{array}$ & 40 & 32 & no aplicable \\
\hline $\begin{array}{l}\text { Inmovilizado intangible } \\
\text { Patente }\end{array}$ & 360 & 400 & \\
\hline $\begin{array}{l}\text { Total UGE } \\
\text { Total valor recuperable UGE }\end{array}$ & 980 & no aplicable & \\
\hline Ajuste & 600 & & \\
\hline
\end{tabular}

\section{Solución.-}

Asignación de la pérdida por deterioro de valor de los activos de Fondo:

\begin{tabular}{|l|c|c|c|c|c|}
\hline & $\begin{array}{c}\text { Importe } \\
\text { en libros }\end{array}$ & $\begin{array}{c}\text { Precio neto } \\
\text { de venta }\end{array}$ & Ajuste 1 $^{\mathbf{0}}$ & Ajuste 2 $^{\mathbf{0}}$ & $\begin{array}{c}\text { Importes } \\
\text { ajustados }\end{array}$ \\
\hline Fondo de comercio & 300 & 0 & $(300)$ & & 0 \\
\hline $\begin{array}{l}\text { Inmovilizado material } \\
\text { Maquinaria } \\
\text { Edificio }\end{array}$ & 40 & 32 & $(8)$ & & 32 \\
\hline $\begin{array}{l}\text { Inmovilizado intangible } \\
\text { Patente }\end{array}$ & 360 & 400 & 0 & & 360 \\
\hline $\begin{array}{l}\text { Total UGE } \\
\text { Total valor recuperable UGE }\end{array}$ & 280 & no aplicable & $(70)$ & $(2)$ & 208 \\
\hline Ajuste & 980 & & & & \\
\hline
\end{tabular}

Deterioro de valor $=380$

1) Fondo de comercio $=300$

2) Reparto restante (80 um) entre maquinaria y patente, ya que sobre el edificio no procede ajuste al ser su precio de venta superior

- Maquinaria: $80 \times \frac{40}{40+280}=10$ 
pero dado que 40-32 = 8, sólo se reduce en 8 porque no se puede reducir el valor del elemento por debajo de su precio de venta.

- Patente: $80 \times \frac{280}{40+280}=70$

El resto de la pérdida se asigna también a la patente (2).

Registro contable:

Por el deterioro del fondo de comercio:

\begin{tabular}{|c|c|}
\hline $\begin{array}{l}00690 \text { Pérdidas por deterioro del } \\
\text { inmovilizado intangible }\end{array}$ & 204 Fondo de comercio \\
\hline
\end{tabular}

Por el deterioro de valor de los activos identificables:

\begin{tabular}{c}
891 Pérdidas por deterioro del \\
$\begin{array}{c}\text { inmovilizado material } \\
72\end{array}$ \\
$\begin{array}{l}690 \text { Pérdidas por deterioro del } \\
\text { inmovilizado intangible }\end{array}$ \\
\hline
\end{tabular}

\begin{tabular}{|c|}
\hline $\begin{array}{l}291 \text { Deterioro de valor del } \\
\text { inmovilizado material }\end{array}$ \\
\hline $\begin{array}{l}290 \text { Deterioro de valor del } \\
\text { inmovilizado intangible }\end{array}$ \\
\hline
\end{tabular}

Por último, si el fondo de comercio es negativo, se registrará como un ingreso en la cuenta de pérdidas y ganancias "774. Diferencia negativa en combinaciones de negocios".

\section{BIBLIOGRAFÍA}

GONZÁLEZ BeTANCORT, B. (2007) "Ejemplos de aplicación de las normas de registro y valoración aplicables a los elementos de inmovilizado contenidas en el Borrador del PGC", CISS Técnica Contable, núm. 698, pp. 18-29.

HeRnández HernándeZ, F.G. (2008) "El inmovilizado en el nuevo Plan General de Contabilidad. Aspectos resueltos y cuestiones pendientes", Partida doble, núm. 196, pp. 22-35.

INTERNATIONAL ACCOUNTING STANDARDS BOARD (2006) Normas Internacionales de Información Financiera (NIIF), texto completo de las Normas Internacionales de Información Financiera emitidas a 1 de enero de 2006, traducción al español publicada por CISS-PRAXIS con la autorización de la IASCF.

PALLARÉS SANCHIDRIÁN, J. (2007) "Reconocimiento y valoración de los activos intangibles según la NIC 38, Activos Intangibles", CISS Técnica Contable, núm. 702, pp. 47-60. 
Seguí Mas, E. y C. Sarasa Pérez (2006) "La contabilidad de intangibles en las NIC/NIIF: comparativa entre la normativa española y la NIC 38 (2004)", CISS Técnica Contable, núm. 689, pp. 20-27.

ZAMORA RAMíREZ, C. (Coord.) (2007) Análisis práctico y guía de implantación del nuevo PGC. Valencia: CISS. 